\title{
Antihemophilic factor (recombinant) plasma/albumin-free method for the management and prevention of bleeding episodes in patients with hemophilia A
}

\author{
Steven Pipe \\ Department of Pediatrics \\ and Communicable Diseases, University \\ of Michigan, Ann Arbor, MI, USA
}

\begin{abstract}
Hemophilia is a rare genetic bleeding disorder that, if not adequately controlled, is associated with life-threatening bleeding events and serious and costly complications, primarily from joint damage. The advent of effective clotting factor replacement therapy for patients with hemophilia is considered one of the foremost medical advances of the 20th century. The last 3 decades of experience in hemophilia care have witnessed the effectiveness of the care of patients with hemophilia within specialized comprehensive care centers, advances in factor replacement therapies, the benefits of prophylaxis over on-demand replacement therapy, and the role of aggressive management of joint disease to prevent dysfunction. Ongoing challenges, including the management of inhibitors to factor therapies and the consequences of thousands of patients with hemophilia becoming infected with human immunodeficiency virus and hepatitis $\mathrm{C}$ virus in the 1980s from contaminated plasma-derived factor concentrates, have highlighted the need for vigilance with respect to clotting factor product safety, access to care, and a full complement of choice of factor replacement therapies. Advate ${ }^{\mathbb{R}}$ (antihemophilic factor [recombinant] plasma/albumin-free method [rAHF-PFM]) is the first recombinant factor VIII therapy manufactured without human or animal protein additives to eliminate the risk of pathogen transmission that could be carried by these additives. Preclinical studies established bioequivalence with recombinant antihemophilic factor (Recombinate ${ }^{\circledR}$ ), a product with 16 years of clinical experience. Currently licensed in 44 countries worldwide, rAHF-PFM has over 7 years of clinical research within 5 global studies supporting its safety and efficacy in the treatment of patients with hemophilia A.
\end{abstract}

Keywords: factor VIII, hemophilia A, recombinant proteins, clinical trials

\section{Primer on hemophilia therapy Epidemiology}

Hemophilia A or hemophilia B are rare genetically inherited bleeding disorders caused by a deficiency of blood clotting factors VIII (FVIII) or IX, respectively. Hemophilias A and B have an incidence of 1 in 5000 and 1 in 25,000 male births, respectively. Their combined prevalence is estimated to be 13 cases per 100,000 or approximately 17,000 cases in the United States. ${ }^{1}$ Hemophilia A accounts for the great majority $(\sim 85 \%)$ of cases of hemophilia. Disease severity is closely correlated with the level of factor deficiency and can be categorized as mild, moderate, and severe (Table 1); of patients with hemophilia, approximately 9000 have severe disease.

Department of Pediatrics

and Communicable Diseases, University

of Michigan, 1500 East Medical Center

Drive, Ann Arbor, Michigan 48109, USA

Tel +l 7346472893

Fax +l 7349367083

Email ummdswp@med.umich.edu

\section{Clinical impact}

The clinical manifestations of hemophilia are bleeding and bruising, with the extent depending on the severity of the disease. Common sites of bleeding are the joints, soft 
Table I Hemophilia A clinical classification

\begin{tabular}{llll}
\hline & \multicolumn{2}{l}{ Classification } & \\
\cline { 2 - 4 } & Severe & Moderate & Mild \\
\hline $\begin{array}{l}\text { FVIII activity } \\
\text { (\% of normal) }\end{array}$ & $<1 \%$ & $1 \%-5 \%$ & $>5 \%-<40 \%$ \\
$\begin{array}{l}\text { Pattern of bleeding } \\
\text { episodes }\end{array}$ & $24-48$ per year & $4-6$ per year & Uncommon \\
$\begin{array}{l}\text { Cause of bleeding } \\
\text { episodes }\end{array}$ & Spontaneous & Minor trauma & $\begin{array}{l}\text { Major trauma } \\
\text { Surgery }\end{array}$ \\
\hline
\end{tabular}

Adapted from White et al..$^{51}$

tissues and muscles, and to a lesser extent, the mouth, gums, and nose, and the central nervous system. Bleeding in the joints (hemarthrosis) is the most common problem in people with severe hemophilia. Individuals with severe hemophilia may experience frequent recurrent bleeding into their joints or muscles, usually one to two times per week. Those with moderate hemophilia may experience a bleeding episode only once a month, as these patients normally experience a bleeding episode only after injury. In contrast, people with mild hemophilia may experience a bleeding episode less frequently, and usually as a result of surgery or major injury. Uncontrolled or inadequately treated recurrent bleeding into a patient's joints results in severe morbidity and irreversible physical disability (hemophilic arthropathy). ${ }^{2}$ This can result in hospitalization, additional therapy, and, in rare cases, open or arthroscopic synovectomy or joint replacement surgeries. In addition to a risk for mortality and hemarthrosis and arthritis, the risk for serious and life-threatening infections can also complicate the management of hemophilia.

\section{Impact on quality of life}

Another complication of hemophilia is the impact on healthrelated quality of life (HRQOL). In a study of HRQOL, data from patients with hemophilia were compared with normative data. Patients with hemophilia had significantly lower scores on HRQOL measures based on the Short Form 36 Health Survey (eg, physical functioning, bodily pain, general health, and social functioning) as compared with normative data. ${ }^{3,4}$ Specifically, patients with severe hemophilia had lower scores on physical measures compared with normative subjects (physical component scale $[\mathrm{PCS}]=31.9$ vs 52.0, respectively). ${ }^{3}$ In another study evaluating the academic achievement in children with hemophilia, patients with $\leq 11$ bleeding episodes per year achieved higher math (103.6 vs 99.6; $\mathrm{p}=0.030$ ) and total achievement scores (as determined by the Wechsler Individual Achievement Test, 104.4 vs $100.6 ; \mathrm{p}=0.026$ ) than patients with $\geq 12$ bleeding episodes per year. The number of bleeding episodes is also correlated positively with school absenteeism, which may have contributed to a decrease in achievement. ${ }^{5}$

\section{Cost impact}

Treatment for hemophilia, which requires therapy with factor concentrates, can be costly, especially with the advent of new technologies. The annual cost for factor therapy for the treatment of bleeding in severe hemophilia can range from US\$60,000 to greater than US\$150,000 per patient. ${ }^{6}$ However, treatment for the preventable complications of hemophilia can increase health care costs substantially beyond the fixed cost of replacement therapy. ${ }^{7}$ The presence of mild or moderate arthropathy can potentially increase the cost by $146 \%$ and $267 \%$, respectively ( $p<0.01$ compared with no arthropathy). ${ }^{6}$

\section{Treatment}

The primary goal of hemophilia practitioners is to enable patients to live as actively and productively as possible, with minimal complications either from their disease or from any adverse effects associated with therapy. The cornerstone of such therapy is FVIII replacement therapy. The selection of an optimal factor replacement therapy is complex and requires that diverse elements be considered for each individual patient. These include family and individual history, efficacy, inhibitor risk, supply, reimbursement options, patient cost, infusion characteristics that contribute to compliance, and each therapy's vulnerability to blood-borne infectious agents. ${ }^{8}$

\section{Prophylaxis and on-demand treatment regimens}

Treatment for patients with hemophilia requires the infusion of clotting factor derived either from fractionation of human plasma or through recombinant deoxyribonucleic acid (DNA) technology. Plasma-derived FVIII concentrates (pdFVIII) are derived from large pools of human donor plasma while recombinant FVIII (rFVIII) concentrates are produced from mammalian cell lines (eg, Chinese hamster ovary, $\mathrm{CHO}$, cells) genetically altered to express human FVIII. ${ }^{9}$

Replacement therapy with factor concentrates can be administered at home or in a physician's office and can be used prophylactically to prevent bleeding or on-demand to stop bleeding when it occurs. The number of infusions required for each bleed and, over time, the number of units of clotting factor needed per infusion, depends on the severity and location of the bleed, the severity of overall disease, and the patient's weight. Prophylactic therapy is most often 
used for people with severe hemophilia, and can be given on a long-term basis or on a shorter-term basis, lasting a few months. Prophylaxis commonly involves single or multiple weekly infusions when there is no acute bleeding, in order to prevent bleeds and cumulative musculoskeletal damage. On-demand therapy is less intensive and is commonly used for people with mild or moderate hemophilia, or for those patients with severe disease who are unable to sustain prophylactic therapy.

Clotting factor therapies exhibit notable interindividual pharmacokinetic variability. ${ }^{10}$ Therefore, it is important that the patient's clinical status, the severity of the disease, and the location and extent of bleeding be reviewed when tailoring FVIII dosing schedules to an individual patient. For on-demand therapy, optimal replacement therapy for acute bleeding should raise plasma levels to $50 \%$ to $100 \%$ of normal for life- or limb-threatening bleeds, and to $30 \%$ to $50 \%$ for lesser bleeds.

Prophylaxis is considered the optimal therapy for children with severe hemophilia. ${ }^{11-13}$ It has proven efficacious in preventing bleeds and joint disease. ${ }^{2}$ This also correlates with a reduction in hospital admissions, emergency department and clinic visits, ${ }^{14}$ and improved academic performance and accomplishments. ${ }^{5,15}$ Additionally, prophylactic regimens may be associated with reduced risk for inhibitor development to factor therapy. ${ }^{16}$ Prophylactic therapy also has been shown to improve HRQOL compared with on-demand therapy..$^{3,4,17}$

\section{Historical safety concerns with factor VIII therapy}

In 1982, the CDC reported a finding that would ultimately change the course of modern medicine. A highly unusual immunosuppressive disorder was detected in 3 patients with hemophilia. ${ }^{18}$ Within 2 years of this report, it is estimated that $63 \%$ of the 15,500 patients living with hemophilia in the United States contracted HIV from plasma-derived clotting concentrates. ${ }^{19}$

Despite epidemiologic evidence at the time that strongly suggested that blood and blood therapies could transmit HIV, opportunities to implement control strategies were seen as "ineffective, too costly, or too risky" by the blood banks, the US Food and Drug Administration, and the plasma fractionation industry. ${ }^{20}$ As detailed in a report issued in 1995 by the Institute of Medicine (IOM), new infections with HIV and fatalities in patients with hemophilia continued to rise while debates among these organizations delayed comprehensive action. Many physicians and health systems continued to urge patients to remain on plasma-derived clotting factor con- centrates. $^{21}$ Sadly, in the absence of effective antiretroviral therapy throughout the early years of the AIDS epidemic, most patients with hemophilia infected with HIV died. ${ }^{22}$ In 1993, the IOM held a series of hearings to provide an objective and impartial review of the decision-making processes and policies that surrounded the contamination of the blood supply with HIV. As a consequence of the hearings, in 1995 the IOM published HIV and the Blood Supply: An Analysis of Crisis Decisionmaking, which offered clear guidelines to preserve and restore the safety of our nation's blood supply and avoid future public health crises. ${ }^{20}$

The significant advances in blood donor screening and blood therapy testing and the adoption of heat-treated factor concentrates reduced the risk for exposure to HIV through blood by the early 1990s. The incidence of HIV transmission in patients with hemophilia fell substantially: the number of HIV-related deaths in the hemophilia population decreased 75\% between 1995 and $1998 .{ }^{20}$ In hemophilia birth cohorts born from 1985 onward, no HIV infection from clotting factor therapies occurred in patients. ${ }^{23}$ The introduction of rFVIII has also had a marked impact; in fact, as a result of recombinant therapy use, no seroconversions to HIV, hepatitis B virus (HBV), or HCV have been reported. ${ }^{24}$ In the United Kingdom, the estimated frequency of infectious HIV donations entering the blood supply from 1996 to 2003 was 0.14 per million. ${ }^{25}$

Although the fear of HIV transmission has largely subsided, the very serious threat posed by new blood-borne pathogens has been recognized. The threat of newly emerging pathogens and concerns over their potential impact on the hemophilia community still remain today. Thus, a key component of hemophilia management is recognition that patients with hemophilia remain vulnerable to potential contamination of the blood supply and clotting factor therapies.

To ensure the safety of the blood supply, 10 tests are now routinely performed for 8 known pathogens on each unit of donated blood in the United States. However, many potentially blood-transmissible pathogens are not currently included in testing protocols. These infectious agents fall into 3 broad categories: (1) known viruses perceived as a low health risk and rarely transmitted, such as hepatitis A; (2) newly identified viruses such as hepatitis G; (3) emerging/spreading viruses and pathogens for which screening procedures have not yet been developed, or whose threats to the blood supply have not yet been assessed or documented. This third category currently includes pathogens with substantial potential for causing illness and even death, such as West Nile virus ${ }^{26}$ and prions. Prions, the etiologic agent 
associated with variant Creutzfeldt-Jakob disease (vCJD), has been defined as one of the significant potential new threats to the blood supply. ${ }^{27}$ First identified in 1996, the impact of vCJD on the clinical practice of hemophilia has been profound, especially given the legacy of HIV and HCV in the hemophilia community. ${ }^{28}$ In the United Kingdom, despite measures to inform all patients about the possible risk for transfusion-transmitted vCJD, many patients' confidence in their therapies has been eroded and some patients fear that their medical and surgical care will be interrupted because of stigmatization, as in the early years of the HIV crisis. These concerns are underscored by a recent study that examined 48 individuals who received a labile blood component from 15 donors who later developed vCJD (55 labile components originating from these 15 donors were issued to hospitals, 48 of these were transfused to recipients, with the remaining 7 components sent to hospitals that were unable to trace their fate)..$^{29}$ One of the recipients received a blood transfusion from a donor who died of pathologically confirmed vCJD 3.5 years after donating blood. The recipient died after developing symptoms of dementia, 6.5 years following the transfusion. Additional cases have been described establishing that the infectious agent can be transmitted by blood components. However, to date there is no evidence that the infectious agent has been transmitted by fractionated plasma components, eg, pdFVIII. ${ }^{30}$ Until a sensitive blood screening test is made available, it is difficult to quantify the absolute risk for additional vCJD transfusion-related cases. For this reason, continued vigilance is important to the response to prion-mediated disease.

The awareness among practitioners that vulnerability to emerging pathogens varies by blood clotting therapy is another important component of hemophilia care. Some rFVIII therapies still incorporate trace amounts of human- or animal-derived albumin. Inactivation techniques are used as part of the processing procedures for all factor therapies that incorporate plasma or plasma additives. Lipid-enveloped pathogens such as HIV, HBV, and $\mathrm{HCV}$ are readily eliminated. However, inactivation measures are only partly or not at all effective in removing or inactivating nonlipid-enveloped viruses, such as human parvovirus B19 (PVB19) and prions. ${ }^{31-33}$ PVB19 has been isolated from plasma-derived coagulation factor concentrates and first-generation rFVIII therapies..$^{34,35}$ However, data suggest that risk for PVB19 transmission by recombinant antihemophilic factor is low. ${ }^{36}$

To address the ongoing concern over potential contamination of blood and blood therapies, national medical councils and physician advisory bodies in the United Kingdom, the United States, Australia, and Canada have independently addressed the need for ongoing vigilance regarding infectious agents in rFVIII replacement therapies. Recently, the UK Haemophilia Centre Doctors' Organisation, the US National Hemophilia Foundation, and the Association of Hemophilia Clinic Directors of Canada have all issued specific guidelines advocating the elimination of albumin from every stage in rFVIII processing. ${ }^{24,37,38}$ The proactive stances taken by these groups, including national health systems, highlight the public support for providing therapies with the least vulnerability to pathogens. According to a report in 2004 by the $\mathrm{CDC}$, the majority of patients with bleeding disorders now use recombinant therapy. ${ }^{39}$ Of nearly 14,000 people registered in the CDC Universal Data Collection surveillance program, $62.7 \%$ of patients with hemophilia A and 56.1\% of patients with hemophilia B used a recombinant therapy in the preceding year.

\section{Development of rAHF-PFM}

Due to the continuing concern within the hemophilia community about potential risks surrounding the use of plasma-derivatives or bovine additives in the cell culture, processing, and formulation of rFVIII, new rFVIII formulations removed human albumin as a stabilizer from the final formulation. However, these "second generation" rFVIII concentrates still may contain residual amounts of protein additives from the cell culture or purification processes. Therefore a "third generation" processing and formulation ultimately resulted in the complete removal of all human- and animal-derived additives from the cell culture process, purification and final formulation and designated recombinant anti-hemophilic factor plasma/ albumin-free method, or rAHF-PFM. The rAHF-PFM process is highly similar to that for the first generation rFVIII, Recombinate ${ }^{\circledR}$ (rAHF), and incorporates: (1) the adaptation of the $\mathrm{CHO}$ cells to new culture conditions free of human- and animal-derived additives; (2) the optimization of the fermentation technology aiming at increased homogeneity and viability of the cells; (3) creation of a new master cell bank; (4) adaptation of the monoclonal antibody production used in the immune affinity purification step to conditions free of human- and animal-derived additives; (5) a dedicated solvent/detergent (S/D) viral inactivation procedure; and (6) a new nonsucrose containing final formulation principally comprised of mannitol, trehalose, and buffered salts was developed in order to replace the use of albumin as a stabilizer (Table 2). 
Table 2 Production and processing of rAHF-PFM

\begin{tabular}{ll}
\hline Fermentation technology & Continuous chemostat perfusion \\
\hline Cell culture medium & $\begin{array}{r}\text { Protein-free medium } \\
\text { Purification }\end{array}$ \\
& $\begin{array}{r}\text { Immunoaffinity chromatography } \\
\text { in plasma/albumin-free conditions }\end{array}$ \\
& - Cation exchange chromatography \\
& - Anion exchange chromatography \\
Viral inactivation & Solvent/detergent treatment \\
Stabilizer & Trehalose \\
Bulking agent & Mannitol \\
\hline
\end{tabular}

Extensive preclinical testing with $\mathrm{rAHF}-\mathrm{PFM}$ revealed a high degree of biochemical, biophysical, and biological similarity with rAHF. ${ }^{40}$

Since the development of the first rFVIII, a wealth of clinical information has been developed to assess the hemostatic response of standard and modified recombinant clotting factor concentrates for patients in the United States with severe or moderately severe hemophilia A. These therapies include the "first-generation" therapy, Recombinate ${ }^{\circledR}$ (antihemophilic factor [recombinant]) (rAHF); the "second-generation" therapies, Kogenate ${ }^{\circledR}$ FS (antihemophilic factor [recombinant]) (KFS), and ReFacto ${ }^{\circledR}$ (antihemophilic factor [recombinant]) (RF) (a B-domain-deleted rFVIII); and "third-generation" recombinant therapy, Advate ${ }^{\circledR}$ (antihemophilic factor [recombinant], plasma/albumin-free method) (rAHF-PFM) (Table 3). Recently, the manufacturing process for RF has been modified to add another third-generation recombinant

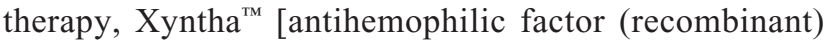
plasma/albumin-free]. To date, there have been no published head-to-head trials of these commonly prescribed therapies for hemophilia.

\section{Efficacy of rAHF-PFM}

To assess the clinical response to therapy, 2 populations of patients with hemophilia are normally studied: previously untreated patients (PUPs) and previously treated patients (PTPs). Factor replacement regimens are evaluated in patients receiving either therapy for prophylaxis of bleeding episodes (including surgery) or on-demand therapy for acute bleeding episodes. Efficacy, as determined by achievement of hemostasis, is evaluated in some studies using a 4-point rating scale. "Excellent" is defined as arrest of bleeding with abrupt pain relief within 8 hours; "Good" is defined as pain relief or reduction of bleeding that was delayed or required more than 1 infusion; "Fair" is defined as a probable or slight beneficial response; "None" is no response. In many trials, patients are monitored for years.

\section{Pharmacokinetics, efficacy, safety and immunogenicity in PTPs}

The Global Clinical Trial Program for rAHF-PFM has included 5 completed clinical trials. ${ }^{40}$ In the Pivotal study ${ }^{41}$ of rAHF-PFM in PTP's $\geq 10$ years of age, the primary aims were to demonstrate the pharmacokinetic properties of rAHF-PFM and to demonstrate safety and efficacy in bleed control and prevention. The study comprised 1) a double-blind, crossover, pharmacokinetic comparison of rAHF and rAHF-PFM, 2) prophylaxis 3 to 4 times per week with 25 to $50 \mathrm{IU} / \mathrm{kg}$ over at least 75 exposure days and 3) treatment of episodic bleeding events. The long-term safety and efficacy of rAHF-PFM was evaluated in 81 subjects from the pivotal study who enrolled in an open-label, two-part continuation study ${ }^{13}$ consisting of 1) a comparison of pharmacokinetics before and after $\geq 75$ exposure days to rAHF-PFM and 2) safety, immunogenicity and hemostatic efficacy of 3 different therapeutic regimens with rAHF-PFM: standard prophylaxis of 25 to $40 \mathrm{IU} / \mathrm{kg}, 3$ to 4 times per week; modified prophylaxis as per the discretion of the investigator; and on-demand therapy. The pharmacokinetics, efficacy, safety and immunogenicity of rAHF-PFM in PTP's less than 6 years of age were evaluated in the pediatric PTP study ${ }^{42}$ wherein subjects were treated according to standard prophylaxis, modified prophylaxis or on-demand therapy. The safety and efficacy of rAHF-PFM in PTPs undergoing surgical, dental or other invasive procedures was assessed in a multicenter, prospective, open-label, uncontrolled surgery study. ${ }^{43}$ Finally a Japanese Registry study ${ }^{13}$ assessed the pharmacokinetics, efficacy, safety and immunogenicity of rAHF-PFM in 15 Japanese PTPs, utilized a study design similar to the Pivotal study, and yielded similar results.

\section{Pharmacokinetic assessment}

Bioequivalence was demonstrated between rAHF-PFM and rAHF based on area under the plasma concentration vs time curve and adjusted recovery following a $50 \pm 5 \mathrm{IU} / \mathrm{kg}$ infusion in a randomized, crossover protocol. Mean $( \pm S D)$ plasma half-life was $11.98 \pm 4.28$ hours. There was substantially less variance in the plasma half-life observed within subjects than among subjects. Pharmacokinetic parameters were similar at the initiation of the trial product and after $\geq 75$ exposure days as assessed in the continuation study.

Within the pediatric study, half-life, adjusted recovery and area under the curve parameters were all lower compared 
to observations within the older subjects in the pivotal trial. Proposed variables affecting the pharmacokinetic parameters include methodologic (pediatric subjects had a delayed first time point of $1 \mathrm{~h}$ post-infusion which is beyond when the peak level was observed in many of the older patients in the Pivotal trial) and physiologic differences (eg, differences in plasma volume to weight ratios, changes in recovery related to body mass index).

\section{Treatment of bleeding episodes}

During the pivotal study, 510 new bleeding episodes in 83 PTP's were treated with rAHF-PFM at a mean dose per infusion of $38.1 \mathrm{IU} / \mathrm{kg}$. Bleeding events were related to trauma $(45 \%)$, spontaneous $(32 \%)$ or undetermined $(24 \%)$. The majority of the bleeds were joints (52\%) or muscles (33\%). Overall, $93 \%$ of the bleeding episodes were resolved with 1 or 2 infusions and $81 \%$ were resolved with only 1 infusion. Patients rated the hemostatic efficacy as excellent or good in $86 \%$ of the bleeding episodes with similar results regardless of the etiology or the anatomic site of bleeding.

In the continuation study, 70 of the 81 subjects reported 822 bleeding episodes, whereas 11 subjects (all of whom were on prophylaxis) reported no bleeding episodes. Among the 820 bleeding episodes that were treated, $88.5 \%$ were managed with one or two infusions.

In the pediatric study, 354 of the 409 reported bleeds were treated with rAHF-PFM. Hemostatic efficacy was rated as excellent or good for $93.8 \%$ of the treated bleeding episodes with $90.1 \%$ requiring only 1 or 2 doses.

\section{Breakthrough bleeding during prophylaxis}

For all patients treated during the first 75 exposure days in the pivotal study, a mean rate of 6.3 new bleeding events per patient per year was observed. However, the annual rate of bleeding varied according to patient adherence to the treatment regimen. Adherence was defined as 25 to $40 \mathrm{IU} / \mathrm{kg}$ per infusion for $>80 \%$ of the prophylactic infusions at a frequency of 3 to 4 times per week for $80 \%$ of the time on study. Adherent patients had a mean annual rate of bleeding of 4.4, whereas less compliant patients had a mean of 9.9 bleeding episodes per year. Similar to the Pivotal trial, subjects who were adherent to the standard prophylaxis regimen in the Continuation study experienced lower annual bleed rates $(\mathrm{n}=30,4.5$ bleeds per year) compared with non-compliant subjects $(\mathrm{n}=24,7.9$ bleeds per year).

Within the pediatric study, the median numbers of annual bleeds for all body sites were 4.0, 4.4 and 24.4 for subjects on standard prophylaxis, modified prophylaxis and on-demand 
therapy respectively. The median annual rate of joint bleeds was zero for all prophylaxis regimens compared to 14.2 for on-demand therapy.

\section{Surgical prophylaxis and perioperative management of hemostasis}

The efficacy and safety of rAHF-PFM in haemophilia patients undergoing surgery was evaluated in a prospective, international, open-label, uncontrolled clinical trial. Patients were $\geq 5$ years of age with baseline FVIII:C $\leq 2 \%$ and $\geq 150$ prior FVIII exposure days. rAHF-PFM was administered perioperatively by bolus infusion (BI) or continuous infusion (CI). The treating surgeon and hematologist both evaluated the efficacy during the hospitalization. Fifty-eight subjects underwent 65 surgical procedures. Twenty-five procedures were considered major hemorrhagic risk, 35 minor and 8 were invasive dental procedures. The hemostatic efficacy was rated as excellent or good for $100 \%$ of the intraoperative and postoperative ratings for both BI and CI modes of administration. Data on estimated blood loss (EBL) were available for 58 procedures. Actual blood loss (ABL) was less than predicted in 27 procedures, within the predicted EBL range for 28 procedures and greater than predicted in 3 procedures. Twelve cases ( 9 of which were major orthopedic surgeries) required transfusion of red blood cells, fresh frozen plasma or albumin. rAHF-PFM was well tolerated in this surgery study with 156 adverse events (AEs) reported in 35 subjects. Seven AEs were serious and 149 non-serious. None of the serious AEs were judged by the investigator to be related to rAHF-PFM, with 8 of the 149 non-serious AEs possibly or probably related. Most AEs were consistent with well-known complications of hemophilia, surgery or hospitalization. There was no difference in AEs among patients receiving $\mathrm{BI}$ or $\mathrm{CI}$ and no thromboembolic complications.

Within the Pediatric study, 7 patients underwent surgical procedures, 5 of which were evaluable per-protocol. These included 2 indwelling port removals, indwelling port removal and circumcision, excision of an ocular cyst and closed reduction of a nasal fracture. ABL and EBL ratios were unremarkable and intraoperative/postoperative hemostatic efficacy was rated as excellent or good in 3 subjects where ratings were available.

\section{Immunogenicity}

One of the most potentially devastating complications for patients with hemophilia is the development of inhibitors (alloantibodies) to FVIII therapy. Inhibitors, measured in Bethesda units (BU), are classified as either high titer ( $>5 \mathrm{BU}$ ) or low titer $(\leq 5 \mathrm{BU})$ and can neutralize the efficacy of treatment.
In addition, inhibitors may often become a barrier to future treatment. Therefore, the prevention of inhibitor development is paramount and a high value is placed on decreasing any risk for developing inhibitors. ${ }^{44}$

The incidence of inhibitors in PTPs is generally low. For example, in the pivotal rAHF trial, none of the 65 patients developed inhibitors after 30 months. ${ }^{45}$ In clinical studies with KFS, no FVIII inhibitors were reported in 71 patients after a mean exposure of 54 days. ${ }^{46}$ The incidence of inhibitor development is similar with RF; only 1 in 113 PTPs treated with RF developed a high titer inhibitor after 113 exposure days ${ }^{47}$ After 18 months of continued therapy, however, this patient's inhibitor level continued to rise and the patient was taken off treatment. In the Pivotal study for rAHF-PFM, 1 of 108 PTPs tested positive for a low titer (2 BU) inhibitor to FVIII following 26 exposure days. The subject was a 55 year old male with severe hemophilia who displayed no symptomatic evidence of an inhibitor. Eight weeks later the inhibitor was undetectable. No inhibitors were detected in the continuation, pediatric or surgery studies.

In contrast to PTPs, inhibitors in PUPs are higher, ranging from $15 \%$ to $33 \%$. In the prospective study of PUPs with rAHF, 22 (31\%) of 72 evaluable patients developed inhibitors. Of these 22 patients, 13 had low titer inhibitors and 9 had high titer inhibitors. ${ }^{48}$ The incidence of inhibitor development was comparable in 101 PUPs treated with RF; with 33 patients (33\%) developing inhibitors. ${ }^{47}$ Of these, 16 patients had high titer inhibitors and 17 had low titer inhibitors. Finally, in an international KFS study in 61 children that also included minimally treated patients, 9 patients $(15 \%$; $9 / 60$; one patient with pre-existing inhibitor was excluded after his 4 th infusion when the result of a positive inhibitor at screening was known) had developed inhibitors ${ }^{49}$ However, many children in this trial remain at risk for developing inhibitors since they have had relatively few exposure days. Clinical trials with rAHF-PFM in PUPs are ongoing. In addition, an ongoing post-licensure safety surveillance study is designed to prospectively assess the incidence of rAHF-PFM-related non-serious adverse events in routine clinical practice and will provide a more accurate estimate of the incidence of inhibitors in a larger hemophilia population within real world practice. ${ }^{13}$

\section{Conclusions}

Remarkable strides in optimizing hemophilia care have been attained over the last several decades. In large part these accomplishments reflect the advent of effective rFVIII therapy, specialized hemophilia treatment centers, and a 
sustained, proactive, long-term decision-making approach that takes into account the unique societal and medical challenges of this disease.

Current studies do not allow an evidence-based decisionmaking tree to determine "best practice" with regards to product choice (ie, recombinant vs plasma-derived products; choice among several generations of recombinant products). Because of the HIV tragedy in the 1980s, the hemophilia community highly values therapies made with processes that minimize risk for pathogen transmission. In dealing with the safety of blood and blood therapies, the precautionary principle is generally followed. This principle posits that complete evidence of risk does not need to exist to take measures to protect against the risk. ${ }^{50}$ Following this principle can conflict with the current practice of evidence-based decision making, which requires that sound evidence of an outcome be produced to justify action. Safety of blood therapies from emerging pathogens is not an outcome that can reasonably be measured in a clinical trial setting, which is the standard setting for collecting data for evidence-based decisions.

rAHF-PFM was developed in response to needs within the hemophilia community for a rFVIII product manufactured without human or animal protein additives to eliminate the risk of pathogen transmission that could be carried by these additives. Preclinical studies established bioequivalence with rAHF and the available clinical data support that it is comparable in terms of safety and efficacy in on-demand and prophylaxis therapy. Within the Global Clinical Study program, 208 PTPs have participated in 5 studies. No subject withdrew due to a rAHF-PFM-related AE, 198 had at least 10 exposure days or 6 months of observation and only 1 subject developed a non-persistent, low-titer inhibitor, and no serious adverse events related to rAHF-PFM have been described.

\section{Disclosures}

SWP has served on Speakers Bureaus for Wyeth, Baxter BioScience and Novo Nordisk.

\section{References}

1. Soucie JM, Evatt B, Jackson D. Occurrence of hemophilia in the United States. The Hemophilia Surveillance System Project Investigators. Am J Hematol. 1998;59:288-294.

2. Manco-Johnson MJ, Abshire TC, Shapiro AD, et al. Prophylaxis versus episodic treatment to prevent joint disease in boys with severe hemophilia. N Engl J Med. 2007;357:535-544.

3. Miners AH, Sabin CA, Tolley KH, et al. Assessing health-related quality-of-life in individuals with haemophilia, Haemophilia. 1999;5:378-385.

4. Royal S, Schramm W, Berntorp E, et al. Quality-of-life differences between prophylactic and on-demand factor replacement therapy in European haemophilia patients. Haemophilia. 2002;8:44-50.
5. Shapiro AD, Donfield SM, Lynn HS, et al. Defining the impact of hemophilia: the Academic Achievement in Children with Hemophilia Study. Pediatrics. 2001;108:E105.

6. Globe DR, Curtis RG, Koerper MA. Utilization of care in haemophilia: a resource-based method for cost analysis from the Haemophilia Utilization Group Study (HUGS). Haemophilia. 2004;10(Supp11):63-70

7. Soucie JM, Nuss R, Evatt B, et al. Mortality among males with hemophilia: relations with source of medical care. The Hemophilia Surveillance System Project Investigators. Blood. 2000;96:437-442.

8. Pipe S. Consideration in hemophilia therapy selection. Semin Hematol. 2006;43:S23-27.

9. Pipe SW. Recombinant clotting factors. Thromb Haemost. 2008; 99:840-850.

10. Shapiro AD, Korth-Bradley J, Poon MC. Use of pharmacokinetics in the coagulation factor treatment of patients with haemophilia. Haemophilia. 2005; 11:571-582.

11. Berntorp E, Boulyjenkov V, Brettler D, et al. Modern treatment of haemophilia. Bull World Health Organ. 1995;73:691-701.

12. Nilsson IM, Berntorp E, Lofqvist T, et al. Twenty-five years' experience of prophylactic treatment in severe haemophilia A and B. J Intern Med. 1992;232:25-32.

13. Shapiro AD. Anti-hemophilic factor (recombinant), plasma/albuminfree method (octocog-alpha; ADVATE) in the management of hemophilia A. Vasc Health Risk Manag. 2007;3:555-565.

14. Panicker J, Warrier I, Thomas R, et al. The overall effectiveness of prophylaxis in severe haemophilia. Haemophilia. 2003;9:272-278.

15. Shapiro AD. A global view on prophylaxis: possibilities and consequences. Haemophilia. 2003;9 Suppl 1:10-17; discussion 18.

16. Gouw SC, van der Bom JG, Marijke van den Berg H. Treatment-related risk factors of inhibitor development in previously untreated patients with hemophilia A: the CANAL cohort study. Blood. 2007;109:4648-4654.

17. Molho P, Rolland N, Lebrun T, et al. Epidemiological survey of the orthopaedic status of severe haemophilia A and B patients in France. The French Study Group. secretariat.haemophiles@cch.ap-hop-paris.fr. Haemophilia. 2000;6:23-32.

18. Pneumocystis carinii pneumonia among persons with hemophilia A. MMWR. 1982;31:365-367.

19. Human immunodeficiency virus infection in the United States: a review of current knowledge. MMWR. 1987;36 Suppl 6:1-48.

20. Committee to Study HIV Transmission Through Blood and Blood Products, Institute of Medicine. HIV and the Blood Supply: An Analysis for Crisis Decisionmaking, National Academy Press, Washington, DC; 1995.

21. Keshavjee S, Weiser S, Kleinman A. Medicine betrayed: hemophilia patients and HIV in the US. Soc Sci Med. 2001;53:1081-1094.

22. Chorba TL, Holman RC, Clarke MJ, et al. Effects of HIV infection on age and cause of death for persons with hemophilia A in the United States. Am J Hematol. 2001;66:229-240.

23. Soucie JM, Richardson LC, Evatt BL, et al. Risk factors for infection with HBV and HCV in a largecohort of hemophiliac males. Transfusion. 2001;41:338-343.

24. National Hemophilia Foundation. MASAC Document \#151: MASAC recommendations concerning the treatment of hemophilia and other bleeding disorders. 2003.

25. Soldan K, Davison K, Dow B. Estimates of the frequency of HBV, $\mathrm{HCV}$, and HIV infectious donations entering the blood supply in the United Kingdom, 1996 to 2003. Euro Surveill. 2005;10:17-19.

26. Pealer LN, Marfin AA, Petersen LR, et al. Transmission of West Nile virus through blood transfusion in the United States in 2002. N Engl J Med. 2003;349:1236-1245.

27. Peden AH, Head MW, Ritchie DL, et al. Preclinical vCJD after blood transfusion in a PRNP codon 129 heterozygous patient. Lancet. 2004;364:527-529.

28. Dolan G. Clinical implications of emerging pathogens in haemophilia: the variant Creutzfeldt-Jakob disease experience. Haemophilia. 2006;12(Suppl 1):16-20; discussion 26-18. 
29. Llewelyn CA, Hewitt PE, Knight RS, et al. Possible transmission of variant Creutzfeldt-Jakob disease by blood transfusion. Lancet. 2004:363:417-421.

30. Turner ML, Ludlam CA. An update on the assessment and management of the risk of transmission of variant Creutzfeldt-Jakob disease by blood and plasma products. Br J Haematol. 2009;144:14-23.

31. Abe H, Sugawara H, Hirayama J, et al. Removal of parvovirus B19 from hemoglobin solution by nanofiltration. Artif Cells Blood Substit Immobil Biotechnol. 2000;28:375-383.

32. Chandra S, Groener A, Feldman F. Effectiveness of alternative treatments for reducing potential viral contaminants from plasma-derived products. Thromb Res. 2002;105:391-400.

33. Knight RS, Will RG. Prion diseases. J Neurol Neurosurg Psychiatry. 2004;75 Suppl 1:i36-i42.

34. Eis-Hubinger AM, Sasowski U, Brackmann HH. Parvovirus B19 DNA contamination in coagulation factor VIII products. Thromb Haemost. 1999;81:476-477.

35. Eis-Hubinger AM, Sasowski U, Brackmann HH, et al. Parvovirus B19 DNA is frequently present in recombinant coagulation factor VIII products. Thromb Haemost. 1996;76:1120.

36. Soucie JM, Siwak EB, Hooper WC, et al. Human parvovirus B19 in young male patients with hemophilia A: associations with treatment product exposure and joint range-of-motion limitation. Transfusion. 2004;44:1179-1185.

37. Guidelines on the selection and use of therapeutic products to treat haemophilia and other hereditary bleeding disorders. Haemophilia. 2003;9:1-23.

38. Association of Hemophilia Clinic Directors of Canada. Clinical Practice Guidelines: Hemophilia and von Willebrand's Disease (2. Management Update 2); 1999.

39. Centers for Disease Control. Report on the Universal Data Collection Program 2004.

40. Ewenstein BM, Collins P, Tarantino MD, et al. Hemophilia therapy innovation: development of an advanced category recombinant factor VIII by a plasma/albumin-free method. Proceedings of a Special Symposium at the XIXth Congress of the International Society on Thrombosis and Haemostasis, July 12-18, 2003, Birmingham, UK. Semin Hematol. 2004;41:1-16; discussion 16-18.

41. Tarantino MD, Collins PW, Hay CR, et al. Clinical evaluation of an advanced category antihaemophilic factor prepared using a plasma/ albumin-free method: pharmacokinetics, efficacy, nd safety in previously treated patients with haemophilia A. Haemophilia. 2004;10:428-437.
42. Blanchette VS, Shapiro AD, Liesner RJ, et al. Plasma and albuminfree recombinant factor VIII: pharmacokinetics, efficacy and safety in previously treated pediatric patients. $J$ Thromb Haemost. 2008;6:1319-1326.

43. Negrier C, Shapiro A, Berntorp E, et al. Surgical evaluation of a recombinant factor VIII prepared using a plasma/albumin-free method: efficacy and safety of Advate in previously treated patients. Thromb Haemost. 2008;100:217-223.

44. Mantovani LG, Monzini MS, Mannucci PM, et al. Differences between patients', physicians' and pharmacists' preferences for treatment products in haemophilia: a discrete choice experiment. Haemophilia. 2005; 11:589-597.

45. White GC 2nd, Courter S, Bray GL, et al. A multicenter study of recombinant factor VIII (Recombinate) in previously treated patients with hemophilia A. The Recombinate Previously Treated Patient Study Group. Thromb Haemost. 1997;77:660-667.

46. Abshire TC, Brackmann HH, Scharrer I, et al. Sucrose formulated recombinant human antihemophilic factor VIII is safe and efficacious for treatment of hemophilia A in home therapy - International KogenateFS Study Group. Thromb Haemost. 2000;83:811-816.

47. Lusher JM, Lee CA, Kessler CM, et al. The safety and efficacy of B-domain deleted recombinant factor VIII concentrate in patients with severe haemophilia A, Haemophilia. 2003;9:38-49.

48. Bray GL, Gomperts ED, Courter S, et al. A multicenter study of recombinant factor VIII (recombinate): safety, efficacy, and inhibitor risk in previously untreated patients with hemophilia A. The Recombinate Study Group. Blood. 1994;83:2428-2435.

49. Kreuz W, Gill JC, Rothschild C, et al. Full-length sucrose-formulated recombinant factor VIII for treatment of previously untreated or minimally treated young children with severe haemophilia A: results of an international clinical investigation. Thromb Haemost. 2005;93:457-467.

50. Wilson K, Ricketts MN. Transfusion transmission of vCJD: a crisis avoided? Lancet. 2004;364:477-479.

51. White GC 2nd, Rosendaal F, Aledort LM, et al. Definitions in hemophilia. Recommendation of the scientific subcommittee on factor VIII and factor IX of the scientific and standardization committee of the International Society on Thrombosis and Haemostasis. Thromb Haemost. 2001;85:560. 
